\title{
A one-act differential stain of the acrosome with active dyes
}

\author{
E. L. Chacarov and M. V. Mollova* \\ Medical Academy, Centre of Oncology, Laboratory of Quantitative Cytology, 6 Plovdivsko pole Ave., \\ Sofia-Darvenitza and \\ *Institute of Biology and Pathology of Reproduction and Non-Infectious Diseases, 73 Lenin Avenue, \\ Sofia 13, Bulgaria
}

Methods of staining the sperm acrosome are of interest because its structural characteristics are an important criterion for assessment of the fertility of ejaculates at fertility clinics and for artificial insemination of domestic animals. The development of deep-freezing techniques for spermatozoa has increased the importance of studying the acrosome because of its sensitivity to cryogenic effects.

The usual sperm stains involve direct dyes which have the disadvantages of being very sensitive to the staining conditions, and are reversible. We have therefore studied some of the so-called active dyes which bind covalently to their substrate. This property is associated with highly reactive atoms or atom groups present in the molecule and the mechanism of action consists of a nucleophile exchange between the basic active group of the dye and the hydroxy or amino-group of the substrate (Karpukhin \& Korotenko, 1963). Reports have been published of attempts to use dyes of this type to give luminescent markers in vitro of the cellular elements of the reticulo-endothelial system in amphibians (Chacarov, Deleva \& Draganov, 1971); for examination of the nervous system (Kater \& Nicholson, 1973; Christensen, 1973); and to study catecholamine derivatives (Sivitz, Kall \& Laties, 1973). Compounds of the chlorotriazine type have been used to fix enzymes to cellulose sheets for industrial purposes (Kay \& Crook, 1967; Kay, Lilly, Sharp \& Wilson, 1968).

\section{Dye screening and staining conditions}

We investigated the staining ability of 15 dyes of the Procion (I.C.I.) group and 18 dyes of the Cibacron (CIBA) group as follows: Procion Green P-5GN, Green 4GS, Purple, Blue HGRS, Brown, Golden Yellow 4RS, Brilliant Red 2RS, Navy Blue, Brilliant Red M8BS, Olive Green M3C3, Printing Green B, Brilliant Red M5BS, Red MX-8B, Blue MRS, and Brilliant Orange M2RS; Cibacron Brilliant Red 2B-E, Brilliant Red 3B-P, Violet 4R-E, Brilliant Grün C4G-A, Blue Brilliant 3R-P, Black FBG-A, Scarlet 2G-E, Brilliant Blue FBR-P, Scarlet R-P, Rubin F2R-P, Brilliant Red 2G-P, Brown 3GR-A, Brilliant Orange G-E, Brilliant Yellow 3G-E, Goldgelb F2R-A, Navy Blue R-E, Green 3G-P, and Brilliant Red 4B-E.

The staining capacity of each dye was studied at concentrations of $0 \cdot 1,1.0$ and $5 \%(\mathrm{w} / \mathrm{v})$; temperature conditions were $18-22^{\circ} \mathrm{C}, 50-55^{\circ} \mathrm{C}$ or $70-80^{\circ} \mathrm{C}$; the $\mathrm{pH}$ of the solution was $1 \cdot 0,3 \cdot 0,5 \cdot 0,7 \cdot 0$, 9.0 or 11.0 ; and the duration of staining $5,10,15,20,30,40$ or $60 \mathrm{~min}$. The staining was performed with either freshly prepared dye solutions or solutions stored for 3,6 or $24 \mathrm{hr}$, because active dyes in an aqueous medium lose their ability to form covalent bonds with their substrate by hydrolysis of the active groups which are substituted for hydroxylic groups.

\section{Experimental material}

Smears of spermatozoa from the following animals were stained: man, bull, ram, boar, rabbit, guinea-pig, rat, hamster, mouse (albino), Apodemus sylvaticus, Apodemus flavicollis, Clethrionomys glareolus and Microtus nivalis. The human spermatozoa and those of bull, ram, boar and rabbit were taken from ejaculates: the spermatozoa were double-washed with $0.9 \% \mathrm{NaCl}$, centrifuged at $1500 \mathrm{~g}$ for $10 \mathrm{~min}$ and resuspended in $0.9 \% \mathrm{NaCl}$. Spermatozoa of the other animals mentioned were obtained by washing the minced caudal part of the epididymis in $0.9 \% \mathrm{NaCl}$. 
Bull, boar and ram spermatozoa were studied both from fresh ejaculates and after equilibration and deep-freezing in liquid nitrogen at $-196^{\circ} \mathrm{C}$ for a period of 30 days to 3 months.

The method of fixation of spermatozoa in the air-dried smears that were tested were as follows: (1) storage at $-10^{\circ} \mathrm{C}$ for $1 \mathrm{hr}$ (unfixed control); (2) passage through a flame; (3) immersion in formalin vapour for 15 or $30 \mathrm{~min}$; (4) immersion in absolute ethyl alcohol for 5,10 or $30 \mathrm{~min}$; (5) immersion in absolute methyl alcohol for 5,10 or $30 \mathrm{~min}$; (6) immersion in a $1: 9$ mixture of glacial acetic acid and absolute ethyl alcohol; and (7) immersion in a $1: 9$ mixture of absolute ethyl alcohol and glacial acetic acid.

The best results for the differential staining of the various structural elements of the spermatozoon and especially the acrosome were obtained with smears fixed for 5-10 min in absolute ethyl alcohol and stained with the dyes Procion Printing Green B and Procion Brilliant Red M8BS under the following conditions: $1 \%$ concentration of the dye, $70-80^{\circ} \mathrm{C}$ temperature, $\mathrm{pH} 1 \cdot 0-3 \cdot 0$ and duration of staining $15-30 \mathrm{~min}$.

The dyes ( $1 \mathrm{~g}$ of each) were dissolved in $100 \mathrm{ml} 1 \%(\mathrm{v} / \mathrm{v})$ aqueous solution of $\mathrm{HCl}$ by heating, and filtered through cottonwool or several layers of gauze. The dye solution was freshly prepared on the day of use because storage leads to de-activation of the dye and reduction of the staining effect. Staining is progressive and the intensity may be controlled by washing the smears in tap water and examining them under the microscope. If necessary the smear can be returned to the dye solution. After a final wash with tap water the smears may be routinely processed or examined dry.

Staining was found to be resistant to subsequent treatment with acid or alkaline organic solvents.

The structural elements of the spermatozoa of all the species studied stained differentially (Plate 1): the acrosome was bright green; the head was pink, the post-nuclear cap being a darker shade of pink; the equatorial segment showed a dense band of pink; the neck showed little colour; the plasmalemma was sometimes visible as a pale greenish contour; the tail stained pale green and was clearly seen; and when present the cytoplasmic droplet was"a dark green.

This differential staining of spermatozoa allows a general morphological characterization of the spermatozoa, and a detailed analysis of the alterations of the acrosome which could arise from disturbances of spermiogenesis or damage during preparation of spermatozoa for deep freezing. The success of the stain, i.e. a common solution of two active dyes at the same $\mathrm{pH}$, may be explained by the presence of an initial phase of electro-affinity binding in which an important role is played by the different electropotentials of the anionogenic auxochrome dye groups and the cationogenic free protein amino-groups. At the very low $\mathrm{pH}$ at which staining takes place, the more weakly dissociating Procion Brilliant Red M8BS stains the highly basic structures of the nuclear protein type while the more strongly dissociating Procion Printing Green B stains the weaker basic structures, mostly in the acrosome but some in the tail.

\section{References}

Chacarov, E., Deleva, N. \& Draganov, A. (1971) Intravital introduction of active dyes into Amphibia. Scr. sci. Med., Varna 9, 15-19.

Christensen, B.N. (1973) Procion brown. An intracellular dye for light and electron microscopy. Science, N.Y. 182, 1255-1256.

Karpukhin, P.P. \& Korotenko, T.A. (1963) Active Dyes. Gos. Izd. Techn. Lit. USSR, Kiev. [Russian].

KATER, S.B. \& Nicholson, C. (Eds) (1973) Intracellular Staining in Neurobiology. Springer, New York.
KAY, G. \& Crook, E.M. (1967) Coupling of enzymes to cellulose using chloro-s-triazines. Nature, Lond. 216, $514-515$.

Kay, G., Lilly, M.D., Sharp, A.K. \& Wilsun, R.J.H. (1968) Preparation and use of porous sheets with enzyme action. Nature, Lond. 217, 641-642.

Sivitz, M., Kall, R.G. \& Laties, A.M. (1973) Procion yellow and catecholamine derivatives: chemical relationships. J. Histochem. 21, 87-92. 
Plate 1
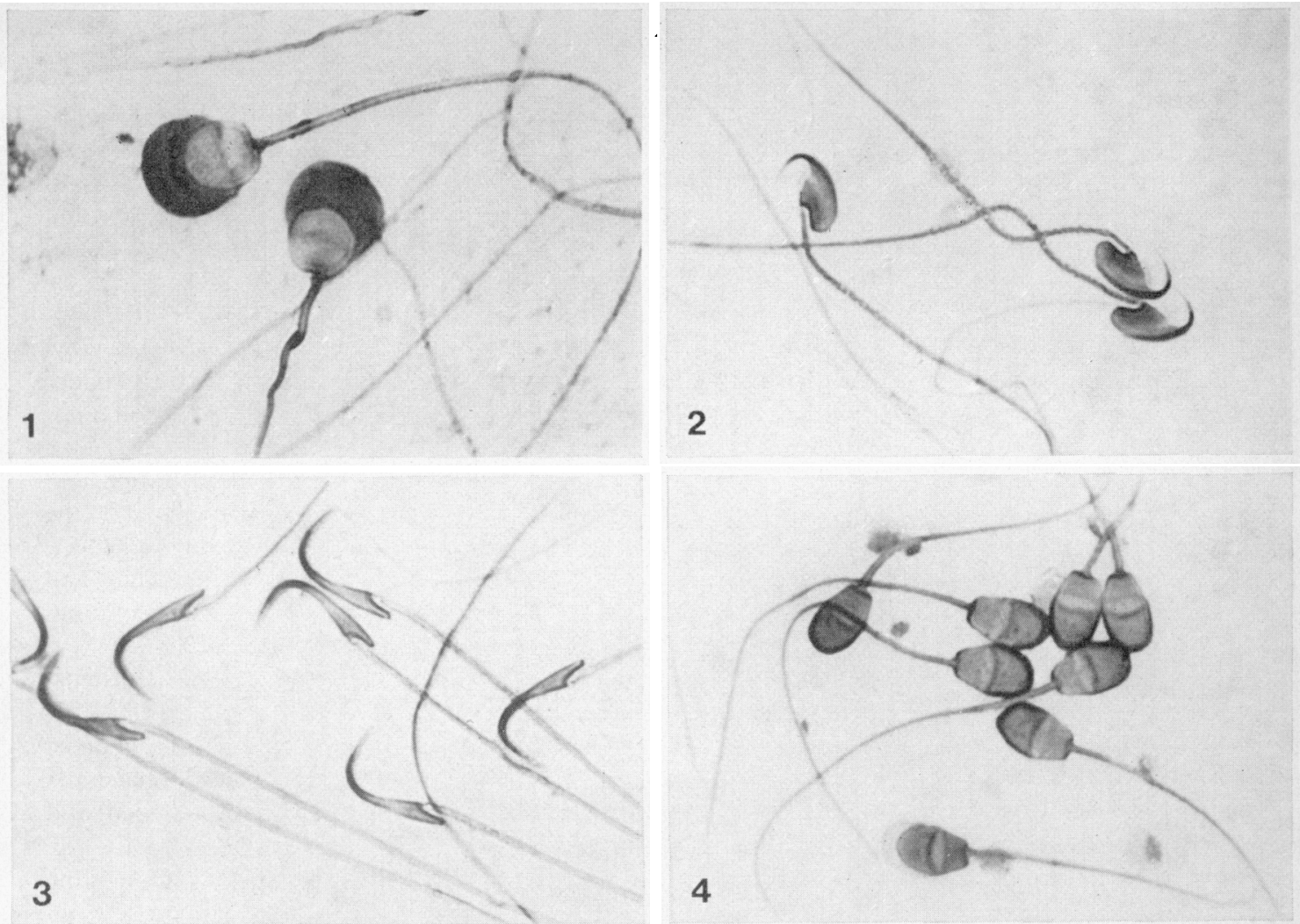

3

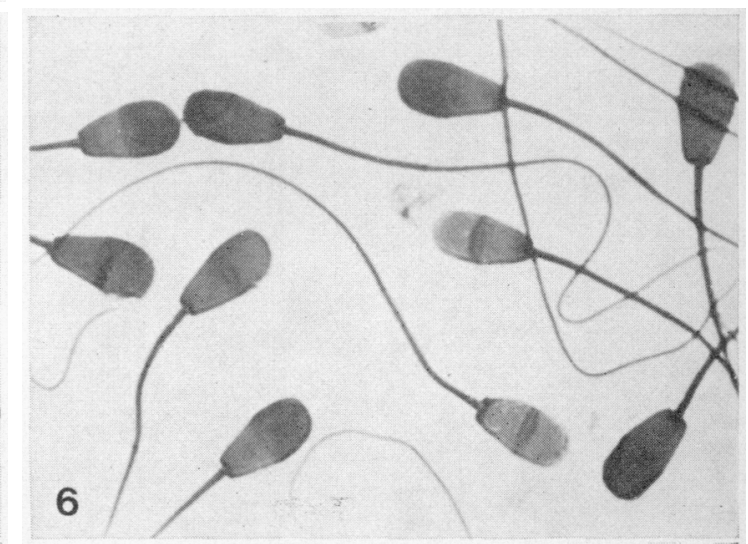

Fig. 1. Guinea-pig epididymal spermatozoa. $\times 410$.

Fig. 2. Epididymal spermatozoa of Apodemus favicollis. Besides the acrosome, the mitochondrial helix is clearly visible. There are residual droplets at the distal end of the middle piece of two of the spermatozoa. $\times 410$.

Fig. 3. Rat epididymal spermatozoa. $\times 410$.

Fig. 4. Ejaculated rabbit spermatozoa. Note the swollen acrosome of the lowest spermatozoon. $\times 410$.

Fig. 5. Ejaculated bull spermatozoa. $\times 410$.

Fig. 6. Ejaculated bull spermatozoa frozen for $\frac{1}{2}$ month at $-196^{\circ} \mathrm{C}$. Note the two spermatozoa without acrosomes; these can be seen near the sperm heads. $\times 410$. 